\title{
Spinal direct current stimulation (tsDCS) in hereditary spastic paraplegias (HSP): A sham- controlled crossover study
}

Gianluca Ardolino, Tommaso Bocci, Martina Nigro, Maurizio Vergari, Alessio Di Fonzo, Sara Bonato, Filippo Cogiamanian, Francesca Cortese, Ilaria Cova, Sergio Barbieri \& Alberto Priori

To cite this article: Gianluca Ardolino, Tommaso Bocci, Martina Nigro, Maurizio Vergari, Alessio Di Fonzo, Sara Bonato, Filippo Cogiamanian, Francesca Cortese, llaria Cova, Sergio Barbieri \& Alberto Priori (2018): Spinal direct current stimulation (tsDCS) in hereditary spastic paraplegias (HSP): A sham-controlled crossover study, The Journal of Spinal Cord Medicine, DOI: 10.1080/10790268.2018.1543926

To link to this article: https://doi.org/10.1080/10790268.2018.1543926

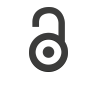

(C) 2018 The Author(s). Published by Informa

UK Limited, trading as Taylor \& Francis Group

曲 Published online: 03 Dec 2018.

Submit your article to this journal $\pi$

III Article views: 412

View Crossmark data \lceil 


\title{
Spinal direct current stimulation (tsDCS) in hereditary spastic paraplegias (HSP): A sham- controlled crossover study
}

\author{
Gianluca Ardolino ${ }^{1}$, Tommaso Bocci ${ }^{1,2,3}$, Martina Nigro', Maurizio Vergari ${ }^{1}$, \\ Alessio Di Fonzo ${ }^{4}$, Sara Bonato ${ }^{4}$, Filippo Cogiamanian", Francesca Cortese ${ }^{1}$, \\ Ilaria Cova ${ }^{5}$, Sergio Barbieri ${ }^{1}$, Alberto Priori ${ }^{4,5}$
}

\begin{abstract}
${ }^{1}$ Neuropathophysiology Unit, Fondazione IRCCS Ca' Granda Ospedale Maggiore Policlinico, Milan, Italy, ${ }^{2}$ Section of Neurophysiopathology, Department of Clinical and Experimental Medicine, Pisa University Medical School, Pisa, Italy, "“Aldo Ravelli” Center for Neurotechnology and Experiental Brain Therapeutics, Department of Health Sciences, University of Milan \& ASST Santi Paolo e Carlo, Milan, Italy, ${ }^{4}$ Neurology Unit, Fondazione IRCCS Ca' Granda Ospedale Maggiore Policlinico, Milan, Italy, ${ }^{5} \mathrm{Clinical}$ Center for Neurostimulation, Neurotechnology, and Movement Disorders, Fondazione IRCCS Ca’ Granda Ospedale Maggiore Policlinico, Milan, Italy
\end{abstract}

Objective: Hereditary spastic paraplegia (HSP) represents a heterogeneous group of neurodegenerative diseases characterized by progressive spasticity and lower limb weakness. We assessed the effects of transcutaneous spinal direct current stimulation (tsDCS) in HSP.

Design: A double-blind, randomized, crossover and sham-controlled study.

Setting: Fondazione IRCCS Cà Granda, Ospedale Maggiore Policlinico, Milan.

Participants: eleven patients with HSP (six men, mean age \pm SD: $37.3 \pm 8.1$ years), eight affected by spastin/ SPG4,1 by atlastin1/SPG3a, 1 by paraplegin/SPG7 and 1 by ZFYVE26/SPG15.

Interventions: tsDCS (anodal or sham, 2.0 mA, 20', five days) delivered over the thoracic spinal cord (T10-T12). Outcome measures: Motor-evoked potentials (MEPs), the H-reflex (Hr), F-waves, the Ashworth scale for clinical spasticity, the Five Minutes Walking test and the Spastic Paraplegia Rating Scale (SPRS) were assessed. Patients were evaluated before tsDCS $\left(T_{0}\right)$, at the end of the stimulation $\left(T_{1}\right)$, after one week $\left(T_{2}\right)$, one month $\left(T_{3}\right)$ and two months $\left(T_{4}\right)$.

Results: The score of the Ashworth scale improved in the anodal compared with sham group, up to two months following the end of stimulation $\left(T_{1}, P=.0137 ; T_{4}, P=.0244\right)$, whereas the Five Minutes Walking test and SPRS did not differ between the two groups. Among neurophysiological measures, both anodal and sham tsDCS left $\mathrm{Hr}$, F-waves and MEPs unchanged over time.

Conclusions: Anodal tsDCS significantly decreases spasticity and might be a complementary strategy for the treatment of spasticity in HSP.

Keywords: Hereditary spastic paraplegias, Movement disorders, Spasticity treatment, Transcutaneous spinal direct current stimulation, Non-invasive spinal stimulation

\section{Introduction}

Hereditary spastic paraplegias (HSP) represent a heterogeneous group of neurodegenerative diseases characterized by progressive spasticity and lower limb weakness, rarely involving all four limbs and variably associated with non-motor symptoms, ranging from sensory

Correspondence to: Alberto Priori, Department of Health Sciences, University of Milan, Via Antonio Di Rudinì 8, 20142 Milan, Italy. Email: mailto:alberto. priori@unimi.it disturbances to cognitive impairment, epilepsy and cerebellar dysfunction. ${ }^{1,2}$ HSP may be inherited by an autosomal or X-linked modality. Approximately 50 loci have been mapped so far; three of them representing approximately $50 \%$ of all mutations, SPG4 being the cause in $40 \%$, SPG3 in $10 \%$ and SPG 31 in $5 \%$ of all families studied. There were no significant associations between disease progression with genotype, but with the age at onset: in particular, for patients with SPG4 
mutations, disease progression was worst in late-onset disease. $^{3}$ From a pathophysiological point of view, both the corticospinal tract axons and ascending lemniscal fibers were impaired. ${ }^{4-6}$ Although the corticospinal tract to the upper limbs is likely spared, ${ }^{7}$ supraspinal disease mechanisms may contribute to the pathology, exerting changes in interhemispheric processing and intracortical excitability. ${ }^{8,9}$ To date, the treatment has been exclusively symptomatic and no conclusive guidelines exist to date. Spasticity may benefit from daily physical therapy, or from baclofen or intramuscular injections of botulinum toxins $\mathrm{A} / \mathrm{B} .^{10}$

Transcutaneous spinal direct current stimulation (tsDCS) has been introduced as a non-invasive technique for modulating spinal cord function in animals and humans. ${ }^{11-19}$ In particular, tsDCS has been used both for interfering with the maladaptive phenomena taking place in spinal cord-injured patients ${ }^{20}$ and improving the effects of robotic gait-training in chronic stroke patients. ${ }^{21}$ Interestingly, although the mechanisms of action have only partly been elucidated, they likely rely both on spinal and supraspinal targets, thus possibly modulating GABA(a)ergic intracortical networks and interhemispheric balance. ${ }^{22-24}$ Different from transcranial direct current stimulation (tDCS), anodal tsDCS has probably an overall inhibitory effect on spinal cord activity: in particular, anodal polarization could act directly on corticospinal descending pathways, without changes in postsynaptic motor neuronal excitability, while cathodal stimulation seems to interfere with interneuronal networks. ${ }^{11-14}$

The aim of this study was to evaluate the effects of tsDCS on spasticity in patients affected by hereditary spastic paraplegias. We mainly focused our attention on spasticity as HSP affects the long tracts with prevalent involvement of the longest upper motor neuron axons, whereas the extension of the damage to the lower motor neuron is extremely variable. To this end, we assessed changes in clinical scores (Ashworth scale, Five Minutes Walking test, Spastic Paraplegia Rating Scale), motor-evoked potentials (MEPs), H-reflex and F-waves in HSP patients who underwent lower thoracic anodal and sham tsDCS.

\section{Materials and methods Subjects}

Eleven patients diagnosed as having HSP were enrolled in the study (six men and five women, mean age \pm SD: $37.3 \pm 8.1$ years); eight were affected by spastin/SPG4 (four men), one man by atlastin1/SPG3a, one woman by paraplegin/SPG7 and one man by ZFYVE26/ SPG15. Each patient signed an informed consent before the inclusion in the study. The protocol was approved by the local ethical committee, in accordance with the tenets of the Declaration of Helsinki. The exclusion criteria were pregnancy, skin lesions of the stimulation area, pacemakers or other electronic devices, previous dorsal laminectomy and other neurological disease.

Different neurologists, blinded to the tsDCS condition, performed the screening visit for study enrolment whereas the clinical and electrophysiological examination was performed by two other neurologists, both blinded to the tsDCS condition.

This study follows the CONSORT guidelines, according to the 25-items checklist and to the flow diagram of the statement.

\section{Transcutaneous spinal DC stimulation (tsDCS)}

With participants lying supine on a couch, tsDCS (2.0 $\mathrm{mA}, 20 \mathrm{~min})$ was delivered by a programable stimulator (HDCStim ${ }^{\mathrm{TM}}$, Newronika, Italy) connected to a pair of rectangular electrodes, one placed over the spinous process of the tenth thoracic vertebra (from the 10 th to 12 th vertebra, with the major axis parallel to spinal cord) and the other above the right shoulder. ${ }^{16,25}$ The tsDCS electrodes were rectangular pieces of saline-soaked synthetic sponge $(7 \times 8 \mathrm{~cm}$, $56 \mathrm{~cm}^{2}$ on the thoracic spinal cord, and $8 \times 10 \mathrm{~cm}$, $80 \mathrm{~cm}^{2}$ above the right shoulder). We applied a current density of $0.035 \mathrm{~mA} / \mathrm{cm}^{2}$ and delivered a total charge density of $42.8 \mathrm{mC} / \mathrm{cm}^{2}$, below the threshold values for tissue damage. ${ }^{26,27}$ tsDCS polarity referred to the electrode over the spinal cord; by analogy with tDCS, the return electrode was placed over the shoulder, as it reduces interference between anodal and cathodal effects.

At the onset of tsDCS, the current was increased for thirty seconds and, at the offset, it was decreased for thirty seconds in a ramp-like manner, a method shown to achieve a good level of blinding among sessions. For a sham tsDCS, the current was turned on for five seconds and then turned off in a ramp-shaped fashion, thus inducing skin sensations indistinguishable from anodal tsDCS.

Patients were blinded to the tsDCS protocol and did not discriminate between anodal and sham condition. In order to report possible adverse effects, the questionnaire developed by Brunoni and colleagues was administered to each patient. ${ }^{28}$

\section{Clinical scales}

Clinical scores were assessed before and after sham and anodal tsDCS. Changes in the Ashworth scale were 
evaluated (six sub-items: hip extension, leg abduction, knee flexion, knee extension, ankle flexion and ankle extension). The Five Minutes Walking Test (5MWT) and Spastic Paraplegia Rating Scale (SPRS) were also assessed. For 5MWT participants walked back and forth along a 30-m hallway, turning around cones at each end for five minutes. They were allowed to use their habitual assistive devices at each testing session. Walking improvement on the 5MWT is indicated by positive change scores (in meters). SPRS efficiently reflects the severity of functional problems, comprising also non-motor symptoms, and correlates with disease duration. ${ }^{29}$ For both Walking Test and SPRS scores, the best results reached by each patient entered the analysis.

\section{H-reflex}

$\mathrm{H}$ reflex was obtained by delivering rectangular pulses through $\mathrm{Ag}-\mathrm{AgCl}$ electrodes (10-mm diameter) placed over the tibial nerve at the popliteal fossa (interelectrode distance $20 \mathrm{~mm}$ ) and recorded from the soleus muscle through $\mathrm{Ag}-\mathrm{AgCl}$ surface electrodes (10-mm diameter) placed $2 \mathrm{~cm}$ apart over the muscle belly. The hip was kept semiflexed $\left(\sim 110^{\circ}\right)$, the knees slightly flexed $\left(\sim 150^{\circ}\right)$, and the ankles in $\sim 10^{\circ}$ plantar flexion. ${ }^{25}$

The current intensity was gradually enhanced to achieve the H-reflex threshold (the minimum stimulation intensity that evoked a reproducible response higher than $50 \mu \mathrm{V}$ ), maximal $\mathrm{H}$ reflex (Hmax), and maximal compound muscle action potential (CMAPmax). In order to minimize post-activation inhibition, the pulses were delivered at random time points, ranging from 10 to $20 \mathrm{~s}$. Stimulation began at $0-\mathrm{mA}$ intensity and grew in 1-mA steps up to the intensity reaching the maximal $\mathrm{H}$ reflex. ${ }^{25}$ The signals were amplified and bandpass filtered $(3 \mathrm{~Hz}-3 \mathrm{kHz})$. We assessed the H-reflex size (peak-to-peak amplitude, $\mathrm{mV}$ ), and we calculated the Hmax - to - CMAPmax ratio.

\section{F-waves}

$\mathrm{F}$ waves were elicited with a $25 \%$ supramaximal stimulation applied to the tibial nerve and recorded from the AH muscle through a pair of 10-mm surface Ag$\mathrm{AgCl}$ electrodes in a belly-to-tendon configuration. $\mathrm{F}$ waves from the abductor hallucis $(\mathrm{AH})$ muscle were obtained by 20 stimuli delivered to the ankle $(1 \mathrm{~Hz}$ repetition rate). ${ }^{30}$ In order to prove the absence of muscular activity, we recorded the audio EMG feedback from the same muscles used for MEP recording. The F-wave mean latency (milliseconds), minimal latency (milliseconds), mean amplitude $(\mathrm{mV})$, and mean temporal dispersion (milliseconds) were assessed. The filter setting was $15-1500 \mathrm{~Hz}$, and the skin temperature at the ankle kept above $32^{\circ} \mathrm{C}$.

\section{Motor-evoked potentials (MEPS)}

A detailed description of the methods used for MEPs recording has been extensively reported elsewhere. ${ }^{25}$ Transcranial Magnetic Stimulation (TMS) was delivered by a Novametrix Magstim 200 stimulator (Magstim, Whitland, UK) using a flat coil (outer diameter $13.5 \mathrm{~cm}$ ). The coil was ensured in a constant position over the vertex. MEPs were recorded at rest by two standard, non-polarizable $\mathrm{Ag}-\mathrm{AgCl}$ surface electrodes (diameter $10 \mathrm{~mm}$; Technomed Europe), one placed over the belly of the abductor hallucis (AH) muscle and the other on the first metatarsophalangeal joint of the toe. Because the $\mathrm{AH}$ has been used in many TMS studies both in health and disease, ${ }^{31-33}$ we selected this muscle for our experiments. As reported in previous studies, both legs were evaluated separately, beginning from the right side. ${ }^{29}$ The stimulation intensity was kept at $120 \%$ of resting motor threshold (RMT). ${ }^{34,35}$ The threshold was set differently for each side and was analyzed at each time point. The current direction was adjusted, with current flowing in the coil anticlockwise for right $\mathrm{AH}$ and clockwise for the contralateral side. ${ }^{30}$ In order to assess MEP changes among different time points, we delivered the stimulation intensity used at baseline; for the assessment of MEP area and amplitude, the stimulator output was the same at each time point. Eight MEPs were collected at 10-second intervals and averaged at each time point. MEPs were amplified and filtered (bandwidth $3 \mathrm{~Hz}-3 \mathrm{kHz}$; Nicolet Viking IV P). Different variables were studied: RMT (\% of stimulator output), onset latency (milliseconds) and area under the curve ( $\mathrm{mVms}$ ) of the motor response. RMT was measured immediately at the end of stimulation week and at each time interval $\left(T_{2}, T_{3}, T_{4}\right.$; see below); the MEP area and latency were assessed offline on MEPs averaged from eight sweeps.

\section{Experimental design}

Subjects were studied before and after anodal and sham tsDCS. Every patient received both the treatment named anodal (A) or sham (S) session in a cross-over design (AS or SA sequence) To assign patients to the treatment sequence, we used an alternating allocation design. All patients carried out two experimental conditions held at least three months apart, to avoid carry-over effects. Each session, either anodal or sham, lasted five days a week (20' twice a day). The clinical 
scores were assessed by a neurologist, while the electrophysiological recordings were made by a neurologist and technicians and their off-line evaluation by a third examiner, all blinded to the tsDCS polarity. MEPs, $\mathrm{H}-$ reflex and F-waves were derived from both sides. Clinical scores, MEPs, H-reflex and F-waves were assessed before tsDCS $\left(\mathrm{T}_{0}\right)$, immediately at the end of stimulation week (i.e. within few minutes following the final tsDCS session, $\left.T_{1}\right)$, after one week $\left(T_{2}\right)$, one month $\left(T_{3}\right)$ and two months $\left(T_{4}\right)$.

No pharmacological modification was performed during the week of tsDCS and during the three months between the two experimental conditions. Moreover, no other therapeutic interventions (i.e. physical therapy) were performed during the three months.

\section{Statistical analysis}

Values are reported as the mean \pm standard error (S.E.). MEP amplitudes were measured peak-topeak. Nonparametric analyses were used, as all data sets did not pass the Shapiro-Wilk test for normality $(\mathrm{P}<.05)$. tsDCS-induced changes in each variable were then assessed with a Friedman test (non-parametric analysis on paired data) with the main factor "time" (four levels: $T_{1}, T_{2}, T_{3}$ and $T_{4}$ ). In order to disclose significant changes at each time point between anodal and sham tsDCS $\left(T_{1}, T_{2}, T_{3}, T_{4}\right)$, a Wilcoxon matched-pairs signed test was then applied. Both the electrophysiological measures and clinical scores were normalized to baseline before entering the analysis (according to the formula $\left(T_{1}-T_{0}\right) / T_{0} * 100+$ 100). Statistical significance was set at $\mathrm{P}<0.05$. The data were analyzed using SPSS v. 21.0 for Windows (SPSS Inc.).

Table 1 Changes over time in MEP parameters, recorded from right and left side, after tsDCS. Results are reported as mean values \pm S.E.

\begin{tabular}{lcccc}
\hline & \multicolumn{3}{c}{ Motor-evoked potentials } \\
\cline { 2 - 5 } & & $\begin{array}{c}\text { Resting motor } \\
\text { threshold (\% of } \\
\text { stimulator output) }\end{array}$ & $\begin{array}{c}\text { Latency } \\
\text { (ms) }\end{array}$ & $\begin{array}{c}\text { Area } \\
\text { (mVms) }\end{array}$ \\
\hline Anodal & $T_{0}$ & $82.0 \pm 3.0$ & $44.8 \pm 1.2$ & $1.5 \pm 0.3$ \\
(mean \pm SE) & $T_{1}$ & $84.5 \pm 2.7$ & $44.7 \pm 1.1$ & $1.1 \pm 0.2$ \\
& $T_{2}$ & $82.5 \pm 2.9$ & $45.8 \pm 1.3$ & $1.3 \pm 0.1$ \\
& $T_{3}$ & $82.3 \pm 3.1$ & $44.8 \pm 1.4$ & $1.3 \pm 0.2$ \\
Sham & $T_{4}$ & $86.8 \pm 2.1$ & $42.8 \pm 2.2$ & $1.4 \pm 0.2$ \\
(mean \pm SE) & $T_{0}$ & $86.1 \pm 2.3$ & $44.3 \pm 0.8$ & $1.3 \pm 0.2$ \\
& $T_{1}$ & $87.4 \pm 1.9$ & $44.1 \pm 1.0$ & $1.1 \pm 0.1$ \\
& $T_{2}$ & $86.4 \pm 1.9$ & $44.7 \pm 0.9$ & $1.2 \pm 0.1$ \\
& $T_{3}$ & $85.7 \pm 2.1$ & $46.0 \pm 1.2$ & $1.3 \pm 0.2$ \\
& $T_{4}$ & $87.9 \pm 2.1$ & $43.4 \pm 1.2$ & $1.2 \pm 0.2$ \\
\hline
\end{tabular}

\section{Results}

\section{Electrophysiology}

All patients tolerated the procedure well, without adverse effects. They rarely reported a slight tingling or itching sensation below the stimulating electrodes lasting only a few seconds or disappearing just after wetting the electrode sponges.

All patients completed the neurophysiological assessment. Baseline values did not change among the different experimental sessions $(\mathrm{P}>0.2$ for all the comparisons made, Wilcoxon test with "stimulation" as factor).

Anodal tsDCS did not statistically modify the MEP variables when compared to sham group, although the anodal polarization leads to a slight reduction in MEP area over time (Table 1; threshold: $\mathrm{P}=0.23$; onset latency: $\mathrm{P}=0.27$; area: $\mathrm{P}=0.16$, Friedman's test).

None of the remaining electrophysiological measures assessed were significantly changed following tsDCS ( $P>0.35$, Friedman's test, for all the comparisons made: relative raw data are reported in Table 2).

\section{Clinical evaluation}

The baseline values did not change among different experimental sessions ( $\mathrm{P}>0.2$ for all the comparisons made; Table 3).

The score of the Ashworth scale for lower limbs improved in the anodal compared with sham group, in particular up to two months following the end of stimulation, at $T_{1}(\mathrm{P}=0.0137$, Wilcoxon matched-pairs signed test $)$ and $T_{4}(\mathrm{P}=0.0244)$. Among the subitems, patients showed a significant improvement in hip flexion $\left(T_{4}: \mathrm{P}=0.016\right.$, Wilcoxon matched-pairs signed test $)$ and knee extension $\left(T_{2}: \mathrm{P}=0.0156 ; T_{3}\right.$ : $\mathrm{P}=0.0078 ; T_{4}: \mathrm{P}=0.0039$, Fig. 1).

Nine patients only completed the clinical evaluation by performing also the Five Minutes Walking Test and the SPRS. No significant change was found when the scores were compared, although there was a general tendency toward improvement in the anodal group (Five Minutes Walking test: $\mathrm{P}=0.072$, Friedman analysis); no improvement in non-motor symptoms was found, as assessed by the Spastic Paraplegia Rating Scale $(\mathrm{P}=.83)$. the Wilcoxon matched-pairs signed test did not reveal significant differences between anodal and sham tsDCS at any time point $(\mathrm{P}>.1)$.

\section{Discussion}

Thoracic tsDCS improved the Ashworth score in HSP patients. To the best of our knowledge, this study is 
Table 2 Changes over time in F-waves and H-reflex parameters between anodal and sham tsDCS. Results are reported as raw data \pm S.E.

\begin{tabular}{|c|c|c|c|c|c|c|c|c|c|}
\hline & & \multicolumn{4}{|c|}{ F-waves } & \multicolumn{4}{|c|}{ H-reflex } \\
\hline & & $\begin{array}{l}\text { Threshold } \\
\text { (mA) }\end{array}$ & $\begin{array}{c}\text { Minimal } \\
\text { latency }(\mathrm{ms})\end{array}$ & $\begin{array}{c}\text { Mean } \\
\text { latency }(\mathrm{ms})\end{array}$ & $\begin{array}{l}\text { Dispersion } \\
\text { (ms) }\end{array}$ & $\begin{array}{l}\text { Threshold } \\
\text { (mA) }\end{array}$ & $\begin{array}{l}\text { Latency } \\
\text { (ms) }\end{array}$ & $\begin{array}{l}\text { Amplitude } \\
\text { (mV) }\end{array}$ & $\begin{array}{c}\text { Ratio } \boldsymbol{H} / \\
\boldsymbol{M}\end{array}$ \\
\hline $\begin{array}{l}\text { Anodal } \\
(\text { mean } \pm \text { SE })\end{array}$ & $\begin{array}{l}T_{0} \\
T_{1} \\
T_{2} \\
T_{3} \\
T_{4} \\
T_{0} \\
T_{1} \\
T_{2} \\
T_{3} \\
T_{4}\end{array}$ & $\begin{array}{l}31.2 \pm 2.7 \\
27.3 \pm 2.2 \\
29.0 \pm 1.9 \\
28.2 \pm 2.5 \\
25.0 \pm 2.0 \\
21.5 \pm 3.7 \\
19.0 \pm 3.0 \\
20.0 \pm 2.1 \\
20.5 \pm 1.8 \\
21.3 \pm 3.8\end{array}$ & $\begin{array}{l}52.2 \pm 1.0 \\
50.0 \pm 1.1 \\
49.7 \pm 0.9 \\
48.9 \pm 1.1 \\
49.0 \pm 0.8 \\
48.1 \pm 0.7 \\
49.5 \pm 1.0 \\
47.3 \pm 0.8 \\
47.7 \pm 0.8 \\
48.3 \pm 0.8\end{array}$ & $\begin{array}{l}54.3 \pm 1.1 \\
52.3 \pm 1.2 \\
51.7 \pm 0.9 \\
51.3 \pm 1.1 \\
51.6 \pm 0.9 \\
50.3 \pm 0.7 \\
51.3 \pm 1.1 \\
50.5 \pm 0.9 \\
50.6 \pm 0.9 \\
50.6 \pm 0.8\end{array}$ & $\begin{array}{l}5.0 \pm 0.5 \\
5.6 \pm 0.4 \\
5.1 \pm 0.4 \\
5.2 \pm 0.3 \\
5.2 \pm 0.4 \\
4.4 \pm 0.3 \\
4.1 \pm 0.4 \\
5.1 \pm 0.3 \\
5.3 \pm 0.7 \\
6.0 \pm 0.6\end{array}$ & $\begin{array}{l}11.3 \pm 0.9 \\
16.2 \pm 1.7 \\
14.0 \pm 1.4 \\
13.0 \pm 1.2 \\
11.5 \pm 1.1 \\
11.8 \pm 0.9 \\
13.0 \pm 1.0 \\
11.2 \pm 0.7 \\
12.2 \pm 0.7 \\
10.2 \pm 0.9\end{array}$ & $\begin{array}{l}30.6 \pm 0.4 \\
30.4 \pm 0.5 \\
29.8 \pm 0.4 \\
29.7 \pm 0.5 \\
29.9 \pm 0.4 \\
30.6 \pm 0.6 \\
28.8 \pm 0.5 \\
29.1 \pm 0.4 \\
28.4 \pm 0.5 \\
28.4 \pm 0.5\end{array}$ & $\begin{array}{l}1.2 \pm 0.6 \\
1.1 \pm 0.7 \\
1.8 \pm 0.4 \\
3.6 \pm 0.6 \\
1.9 \pm 0.5 \\
2.4 \pm 0.7 \\
1.9 \pm 0.6 \\
1.6 \pm 0.4 \\
2.0 \pm 0.5 \\
2.2 \pm 0.6\end{array}$ & $\begin{array}{l}0.1 \pm 0.04 \\
0.2 \pm 0.03 \\
0.2 \pm 0.03 \\
0.3 \pm 0.04 \\
0.2 \pm 0.04 \\
0.3 \pm 0.06 \\
0.2 \pm 0.04 \\
0.2 \pm 0.04 \\
0.3 \pm 0.04 \\
0.2 \pm 0.05\end{array}$ \\
\hline
\end{tabular}

the first to investigate the effects of tsDCS in patients with HSP.

Although tsDCS mechanisms of action still remain debated, a growing body of evidence suggests that tsDCS interferes with cortical, corticospinal, and spinal motor output in humans. ${ }^{36-37}$ Since spinal stimulation modulates both alpha and gamma motor neuron activity in animals, anodal tsDCS could directly inhibit gamma system in humans. ${ }^{14,38}$ In addition, the pre-synaptic inhibition and post-activation depression induced by tsDCS could reduce spasticity by modulating interneuronal excitability. ${ }^{39}$ Spasticity is known to be associated with abnormally weak presynaptic inhibition, as well post-activation depression of synaptic actions of group Ia afferents. ${ }^{39-40}$ Conversely, depending on its polarity, tsDCS may be also useful for the treatment of disease characterized by abnormally strong pre-synaptic inhibition and post-activation depression such as spinal muscular atrophy. ${ }^{40}$

Cortical networks could be additional targets of tsDCS in spasticity. The modulation of cortical

Table 3 Changes in total Ashworth score, 5MWT and SPRS, at baseline and at different time points after tsDCS, following anodal and sham tsDCS. Because the raw data did not assume normality, the results are reported here as mean values and interquartile range (IQR). 5MWT: the 5 Minutes Walking Test; SPRS: Spastic Paraplegia Rating Scale.

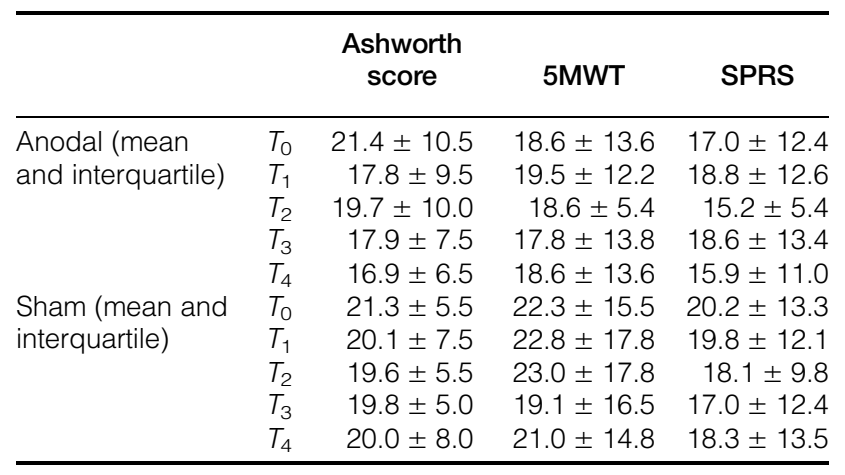

GABAergic activity by tsDCS ${ }^{22}$ could improve spasticity dampening the GABAergic tone, at a spinal as well as cortical level. ${ }^{41-44}$ Further support for a combined cortico-spinal modulation comes from a recent study showing that anodal tsDCS depresses both M1and peripheral-evoked local field potentials (LFP) in the dorsal horn, possibly reducing maladaptive plasticity that contributes to muscle afferent fibers sprouting and hyperreflexia. ${ }^{45}$

Among neurophysiological measures, the lack of changes after tsDCS in the F-waves and H-reflex are in line with those reported in healthy volunteers; ${ }^{25}$ the absence of H-reflex changes suggests no modification in small motor neurons, whereas the lack of F-wave effects rules out changes both in large motor neurons and postsynaptic excitability. ${ }^{46}$

Whereas in healthy subjects anodal tsDCS seemed mainly to affect the RMT and cathodal tsDCS predominantly influenced the MEP area, ${ }^{25}$ in HSP patients anodal polarization left both RMT and MEPs unchanged. This discrepancy might be due to the small and quite heterogeneous sample, but also to the progressive loss of corticospinal fibers compared with controls. Another possibility concerns a ceiling effect obtained in patients with HSP, due to the small number of residual fibers.

The main limitation of our study was the small number of patients enrolled and their genetic pathophysiological heterogeneity. Overall, disease progression significantly differs among various forms, as little progression is seen in some (SPG3a), while a worsening even within few years is described in others (SPG4); ${ }^{3}$ moreover, SPG4 patients only have impaired intracortical excitability and no evidence for supra-spinal disease mechanisms exists so far in SPG3, SPG7 and SPG15. ${ }^{8,9}$ Furthermore, among SPG4 patients, a wide range of genetic mutations have been described so far, from 

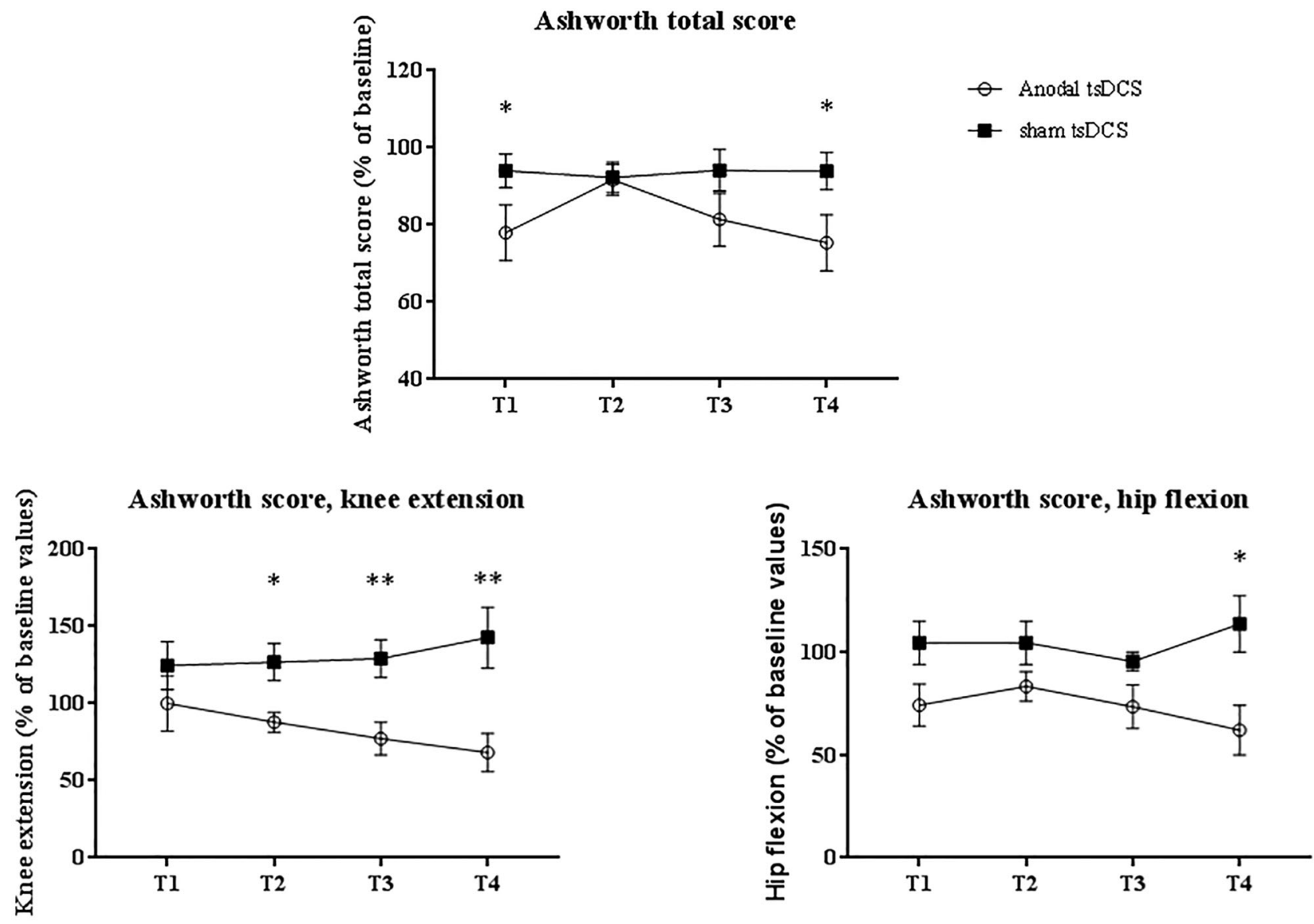

Figure 1 Ashworth scale for lower limbs and sub-items. Anodal tsDCS (white circles) significantly reduced total Ashworth score compared with sham stimulation (black squares), at $T_{1}$ and $T_{4}$; this improvement with respect to hip flexion and knee extension (left and right, bottom row). The data are expressed as mean values \pm S.E. ( ${ }^{*} P<.05 ;{ }^{* *} \mathrm{P}<.01$ ).

large deletions to nonsense and missense mutations, associated with different disease courses. This heterogeneity could explain the relative increase in Ashworth scores at $T_{1}$ and $T_{4}$, as observed in the sham group compared with anodal polarization, due to some patients worsened faster than others. Finally, the effects of direct spinal polarization on non-motor features were not assessed in our study; it is important especially in complex recessive spastic paraplegias, as spatacsin/ SPG11, ZFYVE26/SPG15 and paraplegin/SPG7 ${ }^{47}$ From a statistical point of view, although a crossover study does not require a specific number of patients in each group, it was not possible to counterbalance for eleven patients as this number is not a multiple of the two experimental conditions.

\section{Conclusions}

This study is the first to investigate the effects of tsDCS in patients with HSP. The results are in line with previous data on spinal cord injuries ${ }^{20}$ and chronic stroke, ${ }^{21}$ thereby confirming the efficacy of tsDCS for the treatment of spasticity. Our results may, therefore, contribute to the design of more specific applications in clinical practice; in particular, the use of a combined transcranial and spinal stimulation strategy would be of interest in the wide field of movement disorders, possibly improving motor recovery, as seen in animals. ${ }^{48}$ Moreover, as the improvement lasts up to two month $\left(T_{4}\right)$ with a subsequent worsening of clinical scores, future studies should provide more frequent stimulation cycles. Another critical point to be assessed in future studies is the target of tsDCS; a combined spinal and cortical stimulation protocol to improve the clinical outcome should be evaluated. Lastly, as in other human diseases, the optimal tsDCS repetition rate and duration to promote clinical improvements remains unknown.

\section{Acknowledgements}

The study was supported by Associazione Italiana Vi.P.S. Onlus "Vivere la Paraparesi Spastica". We confirm that we have read the Journal's position on issues involved 
in ethical publication and affirm that this report is consistent with those guidelines. V.M., C.F., S.B. and A.P. are stakeholders in Newronika s.r.l., a spin-off company of the Fondazione IRCCS $\mathrm{Ca}$ ' Granda Ospedale Maggiore Policlinico and the University of Milan.

\section{Disclaimer statements}

Contributors None.

\section{Declaration of interest None.}

Conflicts of interest The authors have no conflict to declare.

Funding The study was supported by Associazione Italiana Vi.P.S. Onlus "Vivere la Paraparesi Spastica". The study was partly supported by POR-FESR 2014 2010 (ELOQUENTSTIM, Regione Lombardia, ID 247367), by donation in memory of Aldo Ravelli, by the Italian Ministry of Health grant (RC-2017 and GR-2011- 02352807) and Roche Research grant 2017.

\section{ORCID}

Sergio Barbieri (D) http://orcid.org/0000-0001-9414-
6307

\section{References}

1 Harding AE. Hereditary spastic paraplegias. Semin Neurol 1993; 13(4):333-6.

2 Lo Giudice T, Lombardi F, Santorelli FM, Kawarai T, Orlacchio A. Hereditary spastic paraplegia: clinical-genetic characteristics and evolving molecular mechanisms. Exp Neurol 2014;261: 518-39.

3 Loureiro JL, Brandao E, Ruano L, et al. Autosomal dominant spastic paraplegias: a review of 89 families resulting from a portuguese survey. JAMA Neurol 2013;70(4):481-7.

4 Blackstone C. Cellular pathways of hereditary spastic paraplegia. Annu Rev Neurosci 2012;35:25-47.

5 Deluca GC, Ebers GC, Esiri MM. The extent of axonal loss in the long tracts in hereditary spastic paraplegia. Neuropathol Appl Neurobiol 2004;30(6):576-84.

6 Orlacchio A, Montieri P, Babalini C, Gaudiello F, Bernardi G, Kawarai $\mathrm{T}$. Late-onset hereditary spastic paraplegia with thin corpus callosum caused by a new SPG3A mutation. J Neurol 2011;258(7):1361-3.

7 Lang N, Optenhoefel T, Deuschl G, Klebe S. Axonal integrity of corticospinal projections to the upper limbs in patients with pure hereditary spastic paraplegia. Clin Neurophysiol 2011;122(7): 1417-20.

8 Nielsen JE, Jennum P, Fenger K, Sorensen SA, FuglsangFrederiksen A. Increased intracortical facilitation in patients with autosomal dominant pure spastic paraplegia linked to chromosome 2p. Eur J Neurol 2001;8(4):335-9.

9 Sartucci F, Tovani S, Murri L, Sagliocco L. Motor and somatosensory evoked potentials in Autosomal Dominant Hereditary Spastic Paraparesis (ADHSP) linked to chromosome 2p, SPG4. Brain Res Bull 2007;74(4):243-9.

10 Geva-Dayan K, Domenievitz D, Zahalka R, Fattal-Valevski A. Botulinum toxin injections for pediatric patients with hereditary spastic paraparesis. J Child Neurol 2010;25(8):969-75.

11 Ahmed Z. Trans-spinal direct current stimulation modulates motor cortex-induced muscle contraction in mice. J Appl Physiol 2011; 110(5):1414-24.
12 Ahmed Z. Effects of cathodal trans-spinal direct current stimulation on mouse spinal network and complex multijoint movements. J Neurosci 2013;33(37):14949-57.

13 Ahmed Z. Electrophysiological characterization of spino-sciatic and cortico-sciatic associative plasticity: modulation by transspinal direct current and effects on recovery after spinal cord injury in mice. J Neurosci 2013;33(11):4935-46.

14 Ahmed Z. Modulation of gamma and alpha spinal motor neurons activity by trans-spinal direct current stimulation: effects on reflexive actions and locomotor activity. Physiol Rep 2016;4(3):e12696.

15 Cogiamanian F, Ardolino G, Vergari M, et al. Transcutaneous spinal direct current stimulation. Front Psychiatry 2012;3:63.

16 Cogiamanian F, Vergari M, Pulecchi F, Marceglia S, Priori A. Effect of spinal transcutaneous direct current stimulation on somatosensory evoked potentials in humans. Clin Neurophysiol 2008; 119(11):2636-40.

17 Cogiamanian F, Vergari M, Schiaffi E, et al. Transcutaneous spinal cord direct current stimulation inhibits the lower limb nociceptive flexion reflex in human beings. Pain 2011;152(2):370-5.

18 Heide AC, Winkler T, Helms HJ, et al. Effects of transcutaneous spinal direct current stimulation in idiopathic restless legs patients. Brain Stimul 2014;7(5):636-42.

19 Priori A, Ciocca M, Parazzini M, Vergari M, Ferrucci R. Transcranial cerebellar direct current stimulation and transcutaneous spinal cord direct current stimulation as innovative tools for neuroscientists. J Physiol 2014;592(16):3345-69.

20 Hubli M, Dietz V, Schrafl-Altermatt M, Bolliger M. Modulation of spinal neuronal excitability by spinal direct currents and locomotion after spinal cord injury. Clin Neurophysiol 2013;124(6):1187-95.

21 Picelli A, Chemello E, Castellazzi P, et al. Combined effects of transcranial direct current stimulation (tDCS) and transcutaneous spinal direct current stimulation (tsDCS) on robot-assisted gait training in patients with chronic stroke: A pilot, double blind, randomized controlled trial. Restor Neurol Neurosci 2015;33(3):357-68.

22 Bocci T, Barloscio D, Vergari M, et al. Spinal Direct Current Stimulation Modulates Short Intracortical Inhibition. Neuromodulation 2015;18(8):686-93

23 Bocci T, Caleo M, Vannini B, et al. An unexpected target of spinal direct current stimulation: Interhemispheric connectivity in humans. J Neurosci Methods 2015;254:18-26.

24 Song W, Amer A, Ryan D, Martin JH. Combined motor cortex and spinal cord neuromodulation promotes corticospinal system functional and structural plasticity and motor function after injury. Exp Neurol 2016;277:46-57.

25 Bocci T, Marceglia S, Vergari M, et al. Transcutaneous spinal direct current stimulation modulates human corticospinal system excitability. J Neurophysiol 2015;114(1):440-6.

26 Liebetanz D, Koch R, Mayenfels S, Konig F, Paulus W, Nitsche MA. Safety limits of cathodal transcranial direct current stimulation in rats. Clin Neurophysiol 2009;120(6):1161-7.

27 McCreery DB, Agnew WF, Yuen TG, Bullara L. Charge density and charge per phase as cofactors in neural injury induced by electrical stimulation. IEEE Trans Biomed Eng 1990;37(10):996-1001.

28 Brunoni AR, Amadera J, Berbel B, Volz MS, Rizzerio BG, Fregni F. A systematic review on reporting and assessment of adverse effects associated with transcranial direct current stimulation. Int J Neuropsychopharmacol 2011;14(8):1133-45.

29 Martinuzzi A, Montanaro D, Vavla M, et al. Clinical and paraclinical indicators of motor system impairment in hereditary spastic paraplegia: a pilot study. PLoS One 2016;11(4):e0153283.

30 Groppa S, Oliviero A, Eisen A, et al. A practical guide to diagnostic transcranial magnetic stimulation: report of an IFCN committee. Clin Neurophysiol 2012;123(5):858-82.

31 Chen R, Tam A, Butefisch C, et al. Intracortical inhibition and facilitation in different representations of the human motor cortex. J Neurophysiol 1998;80(6):2870-81.

32 Nakanishi K, Tanaka N, Fujiwara Y, Kamei N, Ochi M. Corticospinal tract conduction block results in the prolongation of central motor conduction time in compressive cervical myelopathy. Clin Neurophysiol 2006;117(3):623-7.

33 Osei-Lah AD, Mills KR. Optimising the detection of upper motor neuron function dysfunction in amyotrophic lateral sclerosis - a transcranial magnetic stimulation study. J Neurol 2004;251(11): 1364-9. 
34 Di Lazzaro V, Oliviero A, Profice P, et al. Effects of voluntary contraction on descending volleys evoked by transcranial electrical stimulation over the motor cortex hand area in conscious humans. Exp Brain Res 1999;124(4):525-8.

35 Ni Z, Gunraj C, Chen R. Short interval intracortical inhibition and facilitation during the silent period in human. J Physiol 2007;583 (Pt 3):971-82.

36 Murray LM, Tahayori B, Knikou M. Transpinal Direct Current Stimulation produces persistent plasticity in human motor pathways. Sci Rep 2018;8(1):717.

37 Marangolo P, Fiori V, Shofany J, Gili T, Caltagirone C, Cucuzza $\mathrm{G}$, Priori A. Moving beyond the brain: transcutaneous spinal direct current stimulation in post-stroke aphasia. Front Neurol 2017;8:400.

38 Bolzoni F, Jankowska E. Presynaptic and postsynaptic effects of local cathodal DC polarization within the spinal cord in anaesthetized animal preparations. J Physiol 2015;593(4):947-66.

39 Kaczmarek D, Ristikankare J, Jankowska E. Does trans-spinal and local DC polarization affect presynaptic inhibition and postactivation depression? J Physiol 2017;595(5):1743-61.

40 Mentis GZ, Blivis D, Liu W, et al. Early functional impairment of sensory-motor connectivity in a mouse model of spinal muscular atrophy. Neuron 2011;69(3):453-67.
41 Bunday KL, Tazoe T, Rothwell JC, Perez MA. Subcortical control of precision grip after human spinal cord injury. J Neurosci 2014; 34(21):7341-50.

42 Clarkson AN, Huang BS, Macisaac SE, Mody I, Carmichael ST. Reducing excessive GABA-mediated tonic inhibition promotes functional recovery after stroke. Nature 2010;468(7321):305-9.

43 Nardone R, Holler Y, Bathke AC, et al. Spinal cord injury affects Iwave facilitation in human motor cortex. Brain Res Bull 2015;116: 93-7.

44 Blicher JU, Nielsen JF. Cortical and spinal excitability changes after robotic gait training in healthy participants. Neurorehabil Neural Repair 2009;23(2):143-9.

45 Song W, Martin JH. Spinal cord direct current stimulation differentially modulates neuronal activity in the dorsal and ventral spinal cord. J Neurophysiol 2017;117(3):1143-55.

$46 \mathrm{McNeil}$ CJ, Butler JE, Taylor JL, Gandevia SC. Testing the excitability of human motoneurons. Front Hum Neurosci 2013;7:152.

47 Kara E, Tucci A, Manzoni C, et al. Genetic and phenotypic characterization of complex hereditary spastic paraplegia. Brain 2016;139:1904-18

48 Song W, Truong DQ, Bikson M, Martin JH. Transspinal direct current stimulation immediately modifies motor cortex sensorimotor maps. J Neurophysiol 2015;113(7):2801-11. 